\title{
Evolution of the charge localization process in xenon cluster ions: From tetramer to dimer cores as a function of cluster size
}

\author{
T. Laarmann \\ Hamburger Synchrotronstrahlungslabor HASYLAB at Deutsches Elektronen Synchrotron DESY, Notkestrasse \\ 85, D-22603 Hamburg, Germany
}

A. Kanaev

LIMHP CNRS, Université Paris-Nord, Avenue J. B. Clément, 43930 Villetaneuse, France

K. von Haeften, H. Wabnitz, R. von Pietrowski, and T. Möller

Hamburger Synchrotronstrahlungslabor HASYLAB at Deutsches Elektronen Synchrotron DESY, Notkestrasse 85, D-22603 Hamburg, Germany

(Received 21 February 2001; accepted 30 January 2002)

\begin{abstract}
The charge localization process in $\mathrm{Xe}_{N}^{+}$cluster ions $(N=40-20000)$ is investigated with fluorescence spectroscopy methods. New discrete and continuous luminescence bands in the visible and near infrared spectral range are observed and are assigned to radiative transitions of ionic dimers, trimers and tetramers inside Xe clusters. The bands are related to the $5 p^{5}{ }^{2} P_{1 / 2}$ $\rightarrow 5 p^{52} P_{3 / 2}$ transition of electronically excited atomic Xe ions and the $6 p \rightarrow 6 s$ transitions of electronically excited Xe neutrals. The dependence of the size of the ionic centers on the Xe cluster size is discussed. In large clusters discrete lines are due to embedded dimer emission and they are identified as $2(1 / 2)_{u} \rightarrow 1(3 / 2)_{g}$ transitions between different vibrational levels. Line positions are blue-shifted by $30( \pm 1) \mathrm{meV}$ with respect to free molecular dimer ions. The energy shift is due to the interaction of the surrounding neutral Xe cluster atoms with the embedded ionic Xe molecules. (C) 2002 American Institute of Physics. [DOI: 10.1063/1.1463441]
\end{abstract}

\section{INTRODUCTION}

The geometrical and electronic structure of neutral and ionic rare gas clusters has attracted considerable interest for many years. Because of their relative simplicity, they can be regarded as model systems for insulators. Neutral rare gas clusters are bound by weak, undirectional van der Waals forces. This situation changes dramatically after ionization. The positive charge tends to delocalize due to interatomic hopping. At the same time the charge induces polarization of the surrounding atoms. These two processes are competitive. The interplay of the kinetic energy of the hole and the interatomic Coulomb energy due to dipole-dipole and monopole-dipole interactions depends on the cluster size and is of fundamental importance for the charge distribution. Whether the charge localizes on a dimer, trimer, tetramer or even larger core is still under discussion, although several detailed investigations have focused on this issue. ${ }^{1,2}$ According to theoretical work the charge delocalization favors the formation of large centers, while the polarization of the surrounding atoms becomes larger if the charge is concentrated. Therefore, one can expect that dimer centers are formed in very large clusters, if we recall that the polarization energy increases with cluster size. For the smallest cluster, the dimer, charge localization takes obviously place on the dimer, while in an intermediate size range trimer and tetramer ions can be formed. An open question in this context is how many atoms are needed, in order to allow a dimer core localization, which is the favored center in bulk rare gas solids. ${ }^{3}$

Many theoretical and experimental results are available on the structure and stability of rare gas cluster ions. ${ }^{4-10}$ Recently, the relaxation dynamics of photoionized Xe clusters and the role of radiative processes in the formation of cluster ions was studied. ${ }^{11}$ As an extension of these studies, we present results obtained by high-resolution fluorescence spectroscopy, which enabled us to analyze the previously observed features in more detail and to observe several new emission bands. In this contribution, we discuss the evolution from tetramer to trimer ionic cores in small cluster ions $(N<100)$ and the merging into dimer centers in the bulk solid. The radiative processes after photoexcitation and the influence of the neutral cluster atoms on the electronic and geometrical structure of $\mathrm{Xe}_{N}^{+}$clusters is investigated. To our knowledge, these are the first experimental results covering a size range up to 20000 atoms, which allows us to follow the charge localization process up to the final dimer core in the bulk solid.

\section{EXPERIMENT}

The measurements were performed at the experimental station CLULU at the synchrotron radiation laboratory HASYLAB in Hamburg. A detailed description is given elsewhere. ${ }^{12}$ In brief, Xe clusters were prepared in a supersonic expansion through conical nozzles (100 and $200 \mu \mathrm{m}$ diam, $15^{\circ}$ and $4^{\circ}$ opening cone angle, respectively). With a stagnation pressure of 300-2500 mbar and a nozzle temperature of $200-300 \mathrm{~K}$ the average cluster size of $N$ atoms per cluster varied between 40 and 20000 . Cluster sizes were determined with the help of well-known scaling laws, ${ }^{13,14}$ 


$$
N=33 \cdot\left(\frac{\Gamma^{*}}{1000}\right)^{2.35} \text { with } \Gamma^{*}=\frac{K \cdot p \cdot d_{\mathrm{eff}}^{0.85}}{T^{2.2875}} .
$$

The width (FWHM) $\Delta N$ of the size distribution is approximately $N$. Monochromatic synchrotron radiation in the energy range between $8-14.5 \mathrm{eV}$ was focused on the cluster beam $5 \mathrm{~mm}$ downstream from the nozzle. The photoexcited Xe clusters emitted fluorescence light, which was detected spectrally undispersed by two different photomultipliers covering the energy range from 8 to $30 \mathrm{eV}$ and 2 to $6 \mathrm{eV}$. For spectrally resolved measurements in the visible and nearinfrared spectral range a commercial 0.275 m CzernyTurner-type monochromator equipped with three gratings (150 1/mm for overview spectra, $1200 \mathrm{l} / \mathrm{mm}$ for high resolution) was used. The spectral resolution with the $1200 \mathrm{l} / \mathrm{mm}$ gratings is $\sim 1 \mathrm{meV}$ and with the $150 \mathrm{l} / \mathrm{mm}$ grating $\sim 10 \mathrm{meV}$ at $1.5 \mathrm{eV}$. The monochromator was coupled to the experimental chamber with a lens system consisting of two LiF/Suprasil-achromats in order to minimize chromatic and spherical aberration. A liquid nitrogen cooled CCD camera attached to the monochromator was used to record the fluorescence spectra. This improved detection system allowed us to record spectra with substantially better quality than in previous measurements. ${ }^{11}$

\section{RESULTS AND DISCUSSION}

Photoexcited $\mathrm{Xe}_{N}^{+}$cluster ions emit broad luminescence bands in the visible and near infrared spectral range. ${ }^{11}$ The main features in the fluorescence spectra $(\hbar \omega=13.48 \mathrm{eV})$ are two groups of cluster-specific bands (I, II) shown in Fig. 1. Kanaev et al. interpreted these emission bands within the Frenkel exciton model assuming the charge to be delocalized on small emitting centers inside the clusters. ${ }^{11}$ The bands are attributed to radiative transitions between spin-orbit split states related to the $5 p^{52} P_{1 / 2} \rightarrow 5 p^{5}{ }^{2} P_{3 / 2}$ transitions of excited atomic Xe ions (see Fig. 3). With increasing cluster size band I is red shifted. The observed variation of the center of the band shows a logarithmic dependence on the cluster size, which is consistent with the Frenkel exciton model. ${ }^{11}$ The intensity variation of band I and II with the cluster size is explained by the change of the cluster temperature. ${ }^{11}$ Increasing cluster size leads to a lower cluster temperature, since the energy released during the ionization process is distributed over the entire cluster. ${ }^{11}$

Due to our improved detection system we can now observe some fine structure in band I and II. Five bands labeled $\mathrm{a}, \mathrm{b}, \mathrm{c}, \mathrm{e}_{1}$, and $\mathrm{e}_{2}$ can be seen. In the spectral range of band I, we observe two overlapping bands. We labeled the bands at $1.92-1.90 \mathrm{eV}$ (a) and at $1.80-1.79 \mathrm{eV}$ (b). Recently performed measurements on $\mathrm{Xe}_{40}$ cluster ions embedded in cold Ne clusters show the splitting of band I in great detail, which is shown in the inset of Fig. 1 and which will be discussed in a forthcoming paper. ${ }^{15}$ Band II contains a continuous band (c) at $1.62 \mathrm{eV}$, which decreases in intensity with increasing cluster size and an additional band $\left(\mathrm{e}_{1}\right)$ at $1.49 \mathrm{eV}$, which becomes dominant for large clusters $(N>400)$. For small clusters $(N<100)$ band $\left(\mathrm{e}_{1}\right)$ has a shoulder $\left(\mathrm{e}_{2}\right)$ on the low energy side located at $1.32 \mathrm{eV}$. Although, the count rate is rather low a sharp line of a vibrational structure of $\left(e_{2}\right)$ can

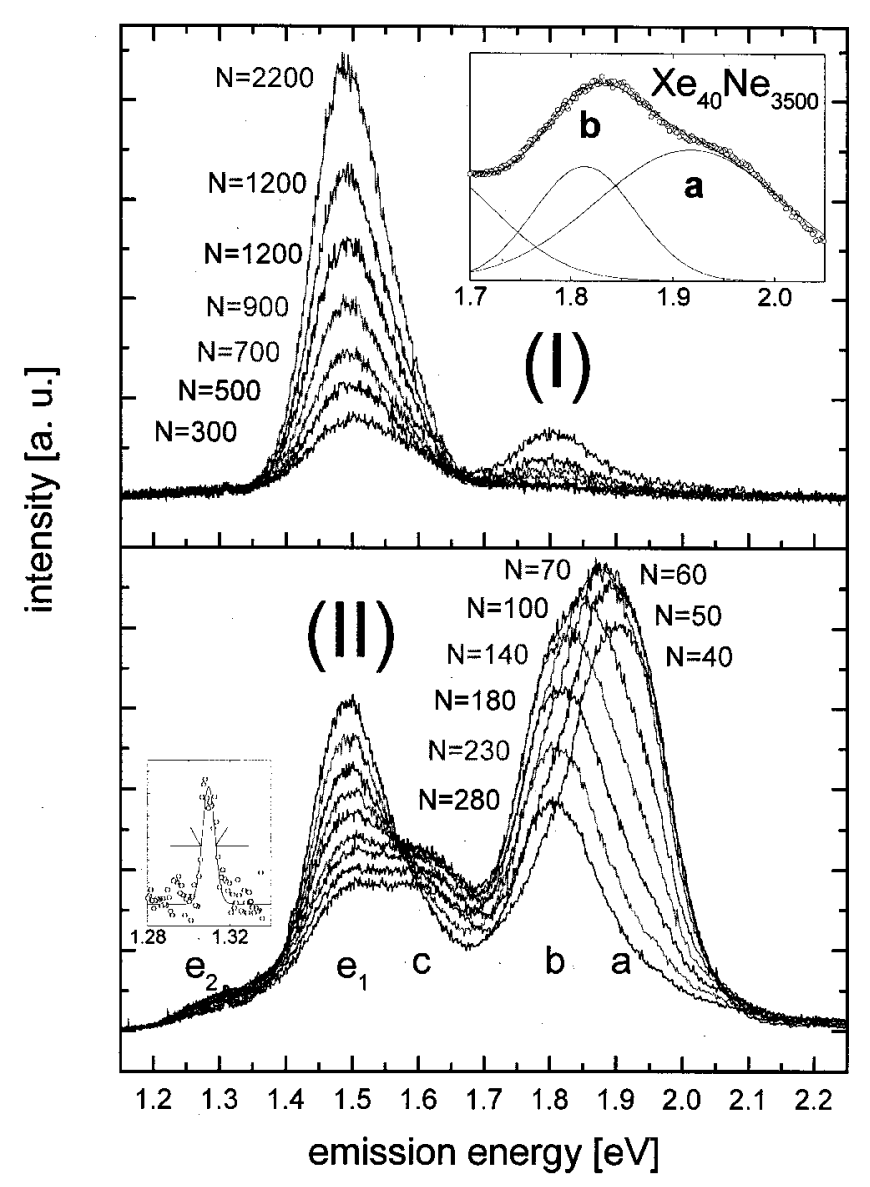

FIG. 1. Fluorescence in the visible and near-infrared spectral range following photoionization of $\mathrm{Xe}_{N}$ clusters (lower part: $40<N<280$ and upper part: $300<N<2200)$ at $13.48 \mathrm{eV}$. The structures (a, b, c, $\mathrm{e}_{1}$, and $\left.\mathrm{e}_{2}\right)$ are related to the $5 p^{5}{ }^{2} P_{1 / 2} \rightarrow 5 p^{5}{ }^{2} P_{3 / 2}$ transition of excited atomic Xe ions and belong to different ionic centers. To show the splitting of band I in more detail the fluorescence spectrum of $\mathrm{Xe}_{40}$ doped $\mathrm{Ne}_{3500}$ clusters is given in the upper inset. In the lower inset a sharp line of the vibrational structure of $e_{2}$ is shown and the experimental resolution is indicated.

be seen in the lower inset of Fig. 1 by subtracting the contribution of band $\left(\mathrm{e}_{1}\right)$ and averaging over a set of spectra. Band $\left(e_{2}\right)$ decreases in intensity with increasing cluster size. The energy positions of band $\mathrm{c}, \mathrm{e}_{1}$, and $\mathrm{e}_{2}$ show no significant variation with cluster size.

High-resolution spectra for large cluster ions excited at $\hbar \omega=13.48 \mathrm{eV}$ are shown in Fig. 2. A vibrational structure of $\mathrm{e}_{1}$ containing many discrete lines is observed. Besides this molecular progression, two intense atomic transitions at $1.506 \mathrm{eV}$ and $1.497 \mathrm{eV}$ are also observed. They are assigned to the $6 p[3 / 2]_{2} \rightarrow 6 s[3 / 2]_{2}$ and $6 p[1 / 2]_{0} \rightarrow 6 s[3 / 2]_{1}$ transitions of neutral Xe atoms. ${ }^{16}$ These unshifted atomic lines indicate that excited $\mathrm{Xe}$ atoms may desorb from the cluster surface during the relaxation process. Direct excitation of Xe atoms into $p$-symmetric states is forbidden because of dipole selection rules. Therefore, we can dismiss the possibility that the lines are due to excited atoms in the background gas, which is always in the beam. The desorption of excited atoms and molecules has recently been observed in light rare gas clusters ( $\mathrm{He}, \mathrm{Ne}$ ) and is obviously an important relaxation channel in these systems. ${ }^{17,18}$ Xe clusters however, show strong desorption and predissociation processes only 


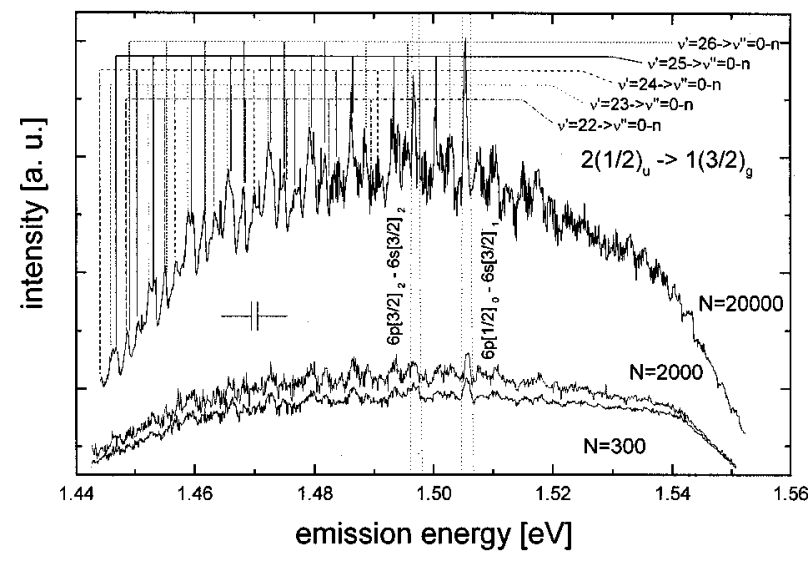

FIG. 2. Fluorescence in the near-infrared spectral range following photoionization of $\mathrm{Xe}_{N}$ clusters $(N=300,2000$, and 20000$)$ at $13.48 \mathrm{eV}$ measured with high spectral resolution. The progression is identified as $2(1 / 2)_{u}$ $\rightarrow 1(3 / 2)_{g}$ transitions of different vibrational levels. The line positions are blueshifted by $30 \mathrm{meV}$ with respect to free molecular Xe ions. The experimental resolution is indicated in the figure.

for small clusters containing less than ten atoms. ${ }^{19}$ Desorption occurs if the interaction between the electronically excited atom and the surrounding atoms is repulsive. Whether the interaction is attractive or repulsive depends on the electron affinity. If the electron affinity is negative, electrons and to some extend electronically excited atoms are repelled by their surrounding. As a result, cavities are formed and desorption takes place once the cavity approaches the surface. ${ }^{20}$ In this context it is important to mention that the electron affinity of Xe clusters varies with size. According to calculations the electron affinity is negative for Xe clusters containing less than $\sim 10$ atoms. ${ }^{21}$ For large clusters it is positive. Thus, desorption is expected to happen only in small $\mathrm{Xe}$ clusters. Small excited $\mathrm{Xe}_{N}$ clusters $(N<10)$ may result from the strong fragmentation process following photoionization. Another possible explanation is the recombination of ions and electrons, which leads to highly excited neutral Xe atoms.

For the interpretation of the progression of discrete lines $\left(\mathrm{e}_{1}\right.$ and $\left.\mathrm{e}_{2}\right)$ and the continuous emission bands $(\mathrm{a}, \mathrm{b}$, and $\mathrm{c})$ one should keep in mind that it is now widely accepted, that after ionization trimer and tetramer centers are formed inside small clusters. Haberland et al. measured photoabsorption cross sections of mass selected $\mathrm{Xe}_{N}^{+}$clusters, $3 \leqslant N \leqslant 30$, and found two distinct families of cluster ions, with either linear trimer or tetramer cores. ${ }^{2}$ In small $\mathrm{Xe}_{N}$ clusters $(N$ $=13,19)$, the ground state charge distribution was expected to be $0.25: 0.5: 0.25$ for a trimer ionic core and $0.1: 0.4$ : $0.4: 0.1$ for a tetramer ionic core, respectively. ${ }^{2}$ The neutral adatoms are arranged in rings of 5 atoms. ${ }^{2}$ There is experimental evidence that the clusters containing less than 30 atoms fluctuate between the two structures on a time scale faster than $10-15$ ns. ${ }^{2}$ However, later calculations by Garcia et al. suggested a tetramer core for $13<N<300$ but with a charge localization of $95 \%$ on one of the four atoms. ${ }^{6}$ These calculations were in good agreement with measurements of the ionization potential of $\mathrm{Xe}_{N}$ clusters. ${ }^{22}$ Charge solvation has also been studied for liquid and solid rare gases. ${ }^{3}$ There

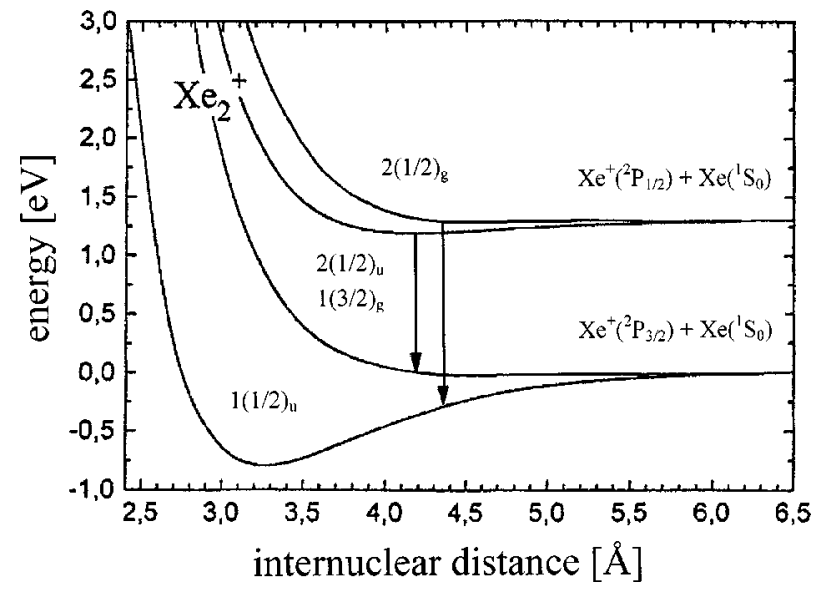

FIG. 3. Schematic potential curves of $\mathrm{Xe}_{2}^{+}$from Ref. 11 including the relevant transitions.

are no optical features in the bulk of solid rare gases because the charged vacancies are neutralised too fast. Nevertheless, if the temperature is low enough to prevent the release of the electrons from their traps in the lattice, the self-trapped holes are stable and can be spectroscopically investigated..$^{23,24}$ So far, it has always been assumed that in the bulk solid localization takes place on a dimer ion. Our measurements can give a deeper insight into charge localization processes in the size range between 40 and 20000 atoms per cluster. For the largest clusters the development of bulk solid properties is expected.

We now assign the emission bands $\mathrm{a}, \mathrm{b}, \mathrm{c}, \mathrm{e}_{1}$, and $\mathrm{e}_{2}$ to dimers, trimers, and tetramers. For the dimer on the basis of $\mathrm{Xe}_{2}^{+}$potential curves shown in Fig. 3 we can only expect two transitions with considerably oscillator strength at $\sim 1.3 \mathrm{eV}$ $\left(2(1 / 2)_{u} \rightarrow 1(3 / 2)_{g}\right)$ and $\sim 1.52 \mathrm{eV}\left(2(1 / 2)_{g} \rightarrow 1(1 / 2)_{u}\right)$. For the analysis we used the spectroscopic constants given in Table I. ${ }^{25}$ If we assume the formation of larger ionic cores than the dimer, potential surfaces have to be considered. Model calculations by Amarouche et al. show that the most stable structures of holes inside small $\mathrm{Xe}_{N}^{+}$clusters $(N$ $<19)$ are linear $\mathrm{Xe}_{3}^{+}$and $\mathrm{Xe}_{4}^{+}$. Trimers have a binding energy of $0.36 \mathrm{eV}$ with respect to the $\mathrm{Xe}_{2}^{+}+\mathrm{Xe}$ asymptote. The energy gain due to hole-delocalization on the third atom is $0.25 \mathrm{eV}$. Polarization forces due to charged induced dipoles lead to a gain of $0.11 \mathrm{eV}$. Tetramers are bound by $0.15 \mathrm{eV}$ with respect to $\mathrm{Xe}_{3}^{+}+\mathrm{Xe}$. In case of $\mathrm{Xe}_{4}^{+}$the calculations give $0.06 \mathrm{eV}$ due to the delocalization energy and $0.09 \mathrm{eV}$ due to the polarization energy. ${ }^{1}$ In these calculations the existence of a stable $\mathrm{Xe}_{5}^{+}$core is excluded because increasing the extent of delocalization is no longer more stabilizing than

TABLE I. Spectroscopic constants for the $2(1 / 2)_{g}, 2(1 / 2)_{u}, 1(3 / 2)_{g}$, and $1(1 / 2)_{u}$ states taken from Ref. 25 .

\begin{tabular}{ccccc}
\hline \hline States & $T_{e}\left(\mathrm{~cm}^{-1}\right)$ & $D_{e}$ & $\omega_{e}$ & $\omega_{e} x_{e}$ \\
\hline $2(1 / 2)_{g}$ & 107500 & 842 & $<30$ & $\ldots$ \\
$2(1 / 2)_{u}$ & 107097 & 1472 & 49.96 & 0.458 \\
$1(3 / 2)_{g}$ & 96208 & 1822 & 58.36 & 0.484 \\
$1(1 / 2)_{u}$ & 90107 & 7923 & 123.41 & 0.486 \\
\hline \hline
\end{tabular}




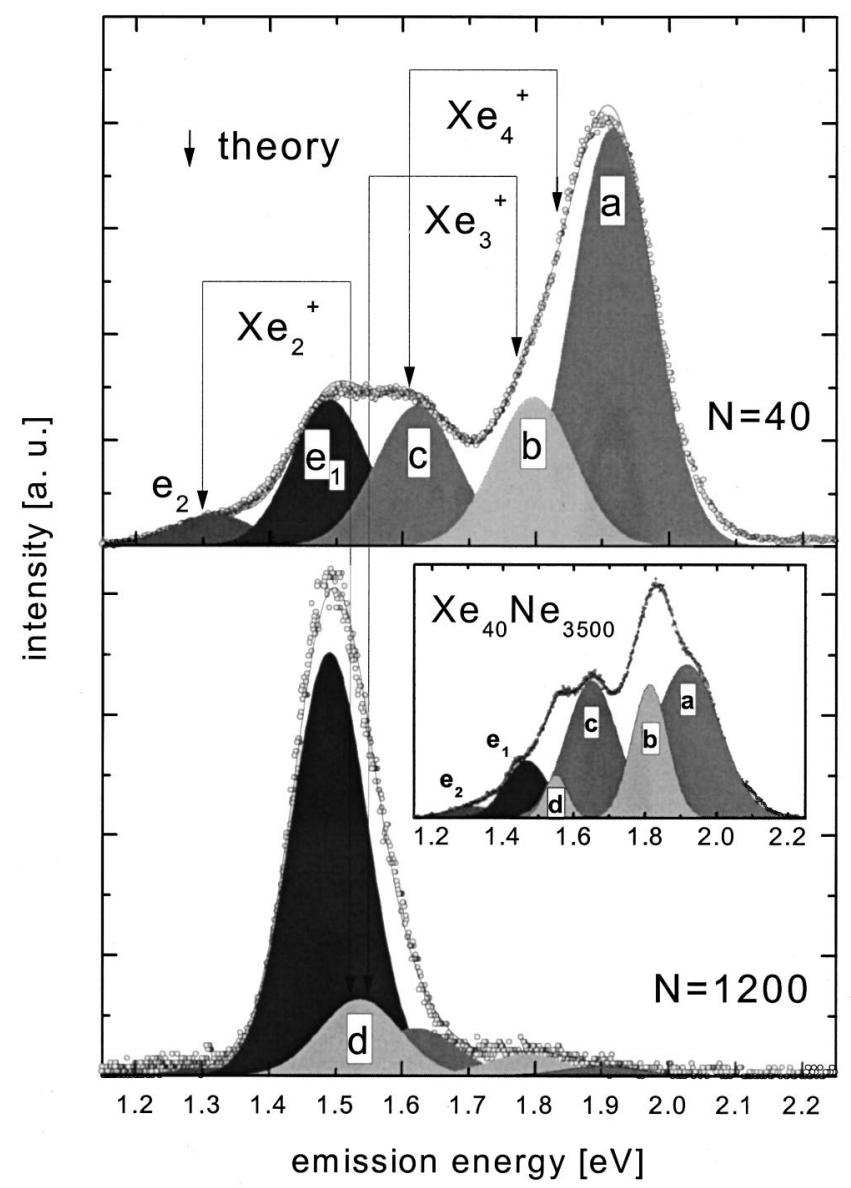

FIG. 4. Fluorescence in the visible and near-infrared spectral range following photoionization of $\mathrm{Xe}_{N}$ clusters $(N=40$ and $N=1200)$ at $13.48 \mathrm{eV}$. The structures (a, b, c, d, $\mathrm{e}_{1}$, and $\mathrm{e}_{2}$ ) are fitted with Gaussian curves. The theoretically estimated dimer, trimer and tetramer transitions are indicated in the figure. To justify the introduction of the additional band (d) the fluorescence spectrum of $\mathrm{Xe}_{40} \mathrm{Ne}_{3500}$ is shown in the inset, where band (d) is observed.

polarizing appropriately positioned atoms around a smaller core. The binding energy of a linear $\mathrm{Xe}_{5}^{+}$center is actually $0.02 \mathrm{eV}$ above the lowest minimum of the tetramer. ${ }^{1}$ The effect of polarization is of similar strength for ground and excited states but only the ground state potential is strongly affected by the stabilization due to charge delocalization. Therefore, we have to consider only delocalization effects in order to estimate the resulting six transition energies of the different ionic subsystems. Corresponding to the dimer transitions at $\sim 1.3 \mathrm{eV}$ and $\sim 1.52 \mathrm{eV}$ and the energy gain due to hole delocalization, we expect the trimer transitions at roughly $1.3+0.25=1.55 \mathrm{eV}$ and $1.52+0.25=1.77 \mathrm{eV}$, respectively. The estimated $\mathrm{Xe}_{4}^{+}$transition energies are therefore $1.55+0.06=1.61 \mathrm{eV}$ and $1.77+0.06=1.83 \mathrm{eV}$, respectively.

In Fig. 4, clusters of around 40 and 1200 atoms are presented. We used six Gaussian-fitting curves to reproduce the spectra. Although, in the fluorescence spectra of pure Xe clusters only five emission bands are clearly observable (a, b, $c, e_{1}$, and $e_{2}$ ), the introduction of band (d) is justified by the above mentioned measurements on embedded $\mathrm{Xe}_{40}$ clusters in cold Ne clusters. The $\mathrm{Xe}_{40} \mathrm{Ne}_{3500}$ spectrum shown in the inset of Fig. 4 enables us to observe band (d) experimentally.
In order to minimize the amount of free parameters, the energy positions for band (c), (d), $\left(e_{1}\right)$, and $\left(e_{2}\right)$ were fixed for all cluster sizes and a logarithmic energy shift of bands (a) and (b) was allowed as already discussed. ${ }^{11}$ The FWHM of the different bands were also kept constant for all cluster sizes: (a, c) $145 \mathrm{meV}$, (b, d) $140 \mathrm{meV}$, and $\left(\mathrm{e}_{1}, \mathrm{e}_{2}\right) 135 \mathrm{meV}$. The theoretically estimated transition energies for the different cores are marked with arrows. The discrepancy of the measured energy positions to the estimated ones is less than $5 \%$. As a result, we assume that band (a) and (b) belong to tetramer and trimer transitions related to $2(1 / 2)_{g}$ $\rightarrow 1(1 / 2)_{u}$. Band (c) and (d) belong to tetramer and trimer transitions related to $2(1 / 2)_{u} \rightarrow 1(3 / 2)_{g}$. The question of whether there is a contribution of dimer transitions $2(1 / 2)_{g}$ $\rightarrow 1(1 / 2)_{u}$ to the intensity of band (d) in large clusters and why the dimer emission $2(1 / 2)_{g} \rightarrow 1(1 / 2)_{u}$ is missing in small clusters will be discussed later. Band $\left(\mathrm{e}_{2}\right)$ belongs to dimer transitions related to $2(1 / 2)_{u} \rightarrow 1(3 / 2)_{g}$. The increasing bandwidth related to $\mathrm{Xe}_{2}^{+}, \mathrm{Xe}_{3}^{+}$, and $\mathrm{Xe}_{4}^{+}$can be explained by the increasing number of different structural isomers with slightly different transition energies, which leads to a broadening of bands. This is in agreement with results of recent investigations on the photofragmentation process in small $\mathrm{Xe}_{4}^{+}$clusters, where experimental evidence for linear and T-shaped isomers was found. ${ }^{26}$ Theoretical studies have revealed many different local minima on the associated potential energy surfaces that correspond to a range of structural isomers. ${ }^{1}$ In cold $\mathrm{Ne}$ and $\mathrm{He}$ clusters $(10 \mathrm{~K}$ and $0.4 \mathrm{~K}$, respectively) structural changes of embedded molecules are suppressed, because of the low temperatures. ${ }^{27}$ Therefore, the number of occurring isomers in doped clusters and the corresponding bandwidth in the fluorescence spectrum is reduced, which enables us to observe the splitting of bands in detail. ${ }^{15}$

Using high-resolution spectroscopy (Fig. 2), we can assign the progression $\left(\mathrm{e}_{1}\right)$ to dimer emissions of $\mathrm{Xe}_{2}^{+}$embedded inside neutral Xe clusters. Using the spectroscopic constants given in Table I, they are identified as $2(1 / 2)_{u}$ $\rightarrow 1(3 / 2)_{g}$ transitions of different vibrational levels $\left(v^{\prime}\right.$ $\left.=22,23,24,25,26, \ldots \rightarrow v^{\prime \prime}=0,1, \ldots\right) .{ }^{25}$ Xenon has nine naturally occurring isotopes with an abundance weighted average mass of $131.3 \mathrm{amu} .{ }^{25}$ It is well known that the shifts in isotopic vibrational energies will become significant at high vibrational level. ${ }^{25}$ Therefore, peaks with high quantum numbers will be broadened and eventually be washed out. Considering this effect, we have calculated the shifts of the $v^{\prime}$ $=26 \rightarrow v^{\prime \prime}=0$ transition taking isotopes into account with a natural abundance of more than $\sim 1 \% .{ }^{28-30}$ We obtain a line broadening of $0.16 \mathrm{meV}$, which is clearly smaller than the spectral resolution at $1.5 \mathrm{eV}(1 \mathrm{meV})$. The line positions are blueshifted by $30( \pm 1) \mathrm{meV}$ with respect to free molecular $\mathrm{Xe}$ ions. The small energy shift is due to an attractive interaction of surrounding matrix atoms on the embedded dimer ion. There is no difference in line position and shape on comparing spectra for $\mathrm{Xe}_{N}$ clusters containing 300-20000 atoms. Taking the icosahedral structure of rare gas clusters with closed shells for $N=13,55,147,309, \ldots$ etc. into account, this is an indication that the interaction is restricted to the first two or three shells of matrix atoms around the ionic 
dimer center. To our knowledge, this is the first example of a discrete vibrational spectrum of an ionic dimer center. One should mention that there is an important difference in the origin of band $\left(\mathrm{e}_{1}\right)$ and $\left(\mathrm{e}_{2}\right)$. Both are related to $2(1 / 2)_{u}$ $\rightarrow 1(3 / 2)_{g}$ transitions of excited ionic dimers, but $\left(\mathrm{e}_{1}\right)$ belongs to partially relaxed dimer ions inside the clusters, while $\left(\mathrm{e}_{2}\right)$ is due to free hot dimer ions. The weak structure of $\left(e_{2}\right)$ shown in the inset of Fig. 1 is due to transitions from very high vibrational levels $\left(v^{\prime}=57 / 58 \rightarrow v^{\prime \prime}=0\right.$ $-n$ solid line). We have to point out, that it is not possible to reproduce the transition energy of $\left(\mathrm{e}_{1}\right)$ with the spectroscopic constants of $\left(e_{2}\right)$ without shifting the potential curves. The matrix-shift is absolutely necessary and gives strong evidence that the partially relaxed dimer ions are inside the cluster.

We assume that the free dimer ions in $2(1 / 2)_{u}$ states with a high degree of internal energy result from fragmentation processes following photoionization of small clusters. This is in principal agreement with photofragmentation data recently presented by Kirkwood et al., in which the kinetic energies of fast neutrals ejected from photoexcited $\mathrm{Ar}_{3}^{+}$was measured and remaining dimer ions with a high degree of internal energy were observed. ${ }^{31}$ One can expect that this observation is also valid for small Xe clusters. For large $\mathrm{Xe}_{N}$ clusters $(N$ $>100)$ the intensity of band $\left(\mathrm{e}_{2}\right)$ decreases because the amount of small clusters in the beam is decreasing. The same argument explains why band (d) for large clusters is mainly due to trimer core transitions and that the contribution of dimers in $2(1 / 2)_{g}$ states is rather low. Band (d) appears for clusters larger than 100 atoms. In this size regime, the amount of small clusters in the beam, which fragment into "hot" dimers is already low. Since the fluorescence spectrum of small clusters $(N=40)$ shows no additional band related to dimer transitions $2(1 / 2)_{g} \rightarrow 1(1 / 2)_{u}$, we assume that the energy redistribution during the fragmentation process leads mainly to free vibrationally excited molecular ions in the lowest electronically excited $2(1 / 2)_{u}$ state. Similar experiments detecting neutral photofragments were carried out for $\mathrm{Ar}_{N}^{+}, \mathrm{Kr}_{N}^{+}$, and $\mathrm{Xe}_{N}^{+}$cluster ions $(2<N<19) .{ }^{32}$ For all three rare gas systems the results show that the primary response to photoexcitation, is the ejection of single atoms with high kinetic energy on a time scale that is short compared with the rotational period of a cluster (10-50 ps) and with the observation time in our experiment $(\sim 1 \mu \mathrm{s}),{ }^{32,33}$

$$
\operatorname{Rg}_{N}^{+}+h \nu \rightarrow \operatorname{Rg}_{N-1}^{+}+\operatorname{Rg}(\text { fast }) .
$$

The fast neutral atoms carry with them up to $50 \%$ of the excess internal energy. ${ }^{34}$ In all cases the remaining internal energy is dissipated by the loss of further neutral fragments with low relative kinetic energies. However, the present experiments show that partially relaxed ionic dimers inside large clusters still exist within the ns-lifetime regime of the excited ionic molecular states.

We now discuss the intensity variation of the different bands with cluster size. In Fig. 5 the measured relative abundance of ionic $\mathrm{Xe}_{2}^{+}, \mathrm{Xe}_{3}^{+}$, and $\mathrm{Xe}_{4}^{+}$cores is displayed as a function of cluster size. The intensities of the bands are normalized to the total fluorescence. In order to neglect the temperature effect on the intensity variation as discussed by Ka-

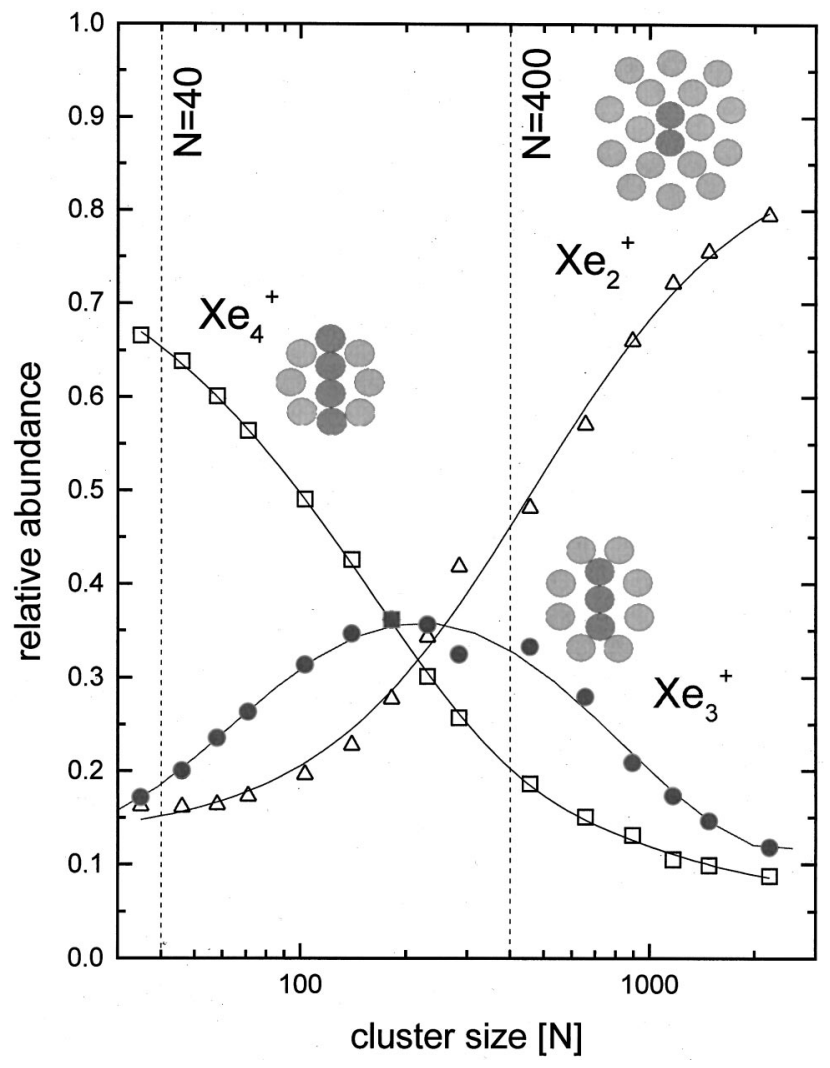

FIG. 5. Relative abundance of dimer, trimer and tetramer ionic centers as a function of cluster size.

naev et al. ${ }^{11}$ we have summed up the intensities of the upper $2(1 / 2)_{g}$ - and lower $2(1 / 2)_{u}$-state belonging to each core. We observe, that in small $\mathrm{Xe}_{N}^{+}$clusters $(N<40)$ the charge is mainly localized on tetramers. The relative abundance is larger than 50\%. In clusters containing more than 40 atoms the relative abundance of tetramer cores continuously decreases, while the charge localization on trimer cores becomes more likely. For an average cluster size of 200 atoms the relative abundance of trimers is at a maximum. In clusters containing more than 200 atoms the relative abundance of trimer cores decreases, while the charge localization on dimer cores becomes more likely. For large cluster ions $(N$ $>400$ ), we observe a merging into bulk properties with the charge localization on mainly dimer cores $(>50 \%)$.

The cluster size dependence of the different ionic cores can be explained in the following way. The charge distribution process depends on the interplay between the interatomic hopping of the hole (charge delocalization) and the polarization of surrounding neutral atoms. The two effects are competitive, since the larger the hole delocalization, the smaller the polarization energy. The equilibrium condition depends on the cluster size, since at least the polarization energy increases with cluster size. ${ }^{35}$ For a quantitative analysis of both effects in Xe clusters the knowledge of the exact atomic structure and the dipole density distribution around the ionic core in the ground and excited states is essential.

In our fluorescence spectra, we can identify five bands and therefore we have the possibility of distinguishing between different cores over a large range of cluster sizes. As 
mentioned above, there is strong evidence that in large $\mathrm{Xe}_{N}$ clusters $(N>400)$ the polarization of the surrounding atoms overcomes the hole delocalization and can cause a compact ionized dimer core to be energetically more favourable. In the following, we try to describe the charge distribution process in a very simple picture, which gives an order of magnitude estimation of these effects. The kinetic energy of the hole is set constant. It is known that the energy of the neutral matrix polarization by the ionic center is slightly larger for dimers than for trimers and tetramers because of the difference in their dimension. ${ }^{6}$ In small $\mathrm{Xe}_{N}^{+}$clusters $(N<13)$ this energy difference is small compared to the dissociation energy of $\mathrm{Xe}_{3}^{+}$and $\mathrm{Xe}_{4}^{+}$. Therefore, in small clusters the formation of $\mathrm{Xe}_{2}^{+}$ionic cores is unlikely because the gain in polarization energy is not sufficient to dissociate the trimer. In large clusters the situation is somewhat different. In this case we estimate the polarization energy in a simple electrostatic model. The $\mathrm{Xe}_{N}^{+}$cluster is treated as a dielectric medium with $(\mathrm{N}-\mathrm{M})$ neutral atoms surrounding the ionic core containing $\mathrm{M}$ atoms. If $n$ is the density of matrix atoms, then each atom has the same volume as a sphere of a radius $R_{N}$ $=[3 /(4 \pi \cdot n)]^{1 / 3}$, therefore the cluster can be approximated by a sphere of $R_{S}=R_{N} N^{1 / 3}$. Concerning the ionic core we approximate $R_{C}=R_{M} M^{1 / 3}$ ( $R_{M}:=$ half of the average interatomic distance for $\mathrm{Xe}_{2}^{+}, \mathrm{Xe}_{3}^{+}$, and $\left.\mathrm{Xe}_{4}^{+}\right)$. Thus, we obtain for the polarization energy of the matrix atoms;

$$
E_{P}=\frac{e^{2}}{2} \cdot\left(1-\varepsilon_{S}^{-1}\right) \cdot\left(\frac{1}{R_{C}}-\frac{1}{R_{S}}\right),
$$

which is the classical Born equation for the solvation of a positive charge inside a dielectric medium. ${ }^{36}$ The average interatomic distances of $\mathrm{Xe}_{2}^{+}, \mathrm{Xe}_{3}^{+}$, and $\mathrm{Xe}_{4}^{+}$are $3.18 \AA$, $3.32 \AA$, and $3.46 \AA .{ }^{1}$ With the solid dielectric constant for xenon $\varepsilon_{S}^{-1}=2.2$, we can calculate the differences in polarization energies for different ionic cores as a function of cluster size. As a result, we obtain for $\mathrm{Xe}_{N}^{+}(N=40): \Delta E_{P}$ $=E_{P}\left(\mathrm{Xe}_{3}^{+}\right)-E_{P}\left(\mathrm{Xe}_{4}^{+}\right) \approx 0.21 \mathrm{eV}$. This is in the range of dissociation energies of the tetramer core $(0.15 \mathrm{eV})$. Therefore, we conclude that in clusters containing more than 40 atoms charge localization on ionic trimers becomes more reasonable because the stabilization due to the polarization energy increases. For $\mathrm{Xe}_{N}^{+}(N=400)$ the calculations give $\Delta E_{P}=E_{P}\left(\mathrm{Xe}_{2}^{+}\right)-E_{P}\left(\mathrm{Xe}_{3}^{+}\right) \approx 0.32$. The dissociation energy of trimers is roughly $0.36 \mathrm{eV}$. Thus, the charge localization on dimer cores is mainly observed for big clusters in agreement with our experimental results.

\section{CONCLUSION}

In this paper we have reported on new discrete and continuous luminescence bands of Xe cluster ions in the visible and near-infrared spectral range. The structures are assigned to radiative transitions of ionic centers inside Xe clusters. The luminescence bands are related to the $5 p^{52} P_{1 / 2}$ $\rightarrow 5 p^{52} P_{3 / 2}$ transition of excited atomic $\mathrm{Xe}$ ions and $6 p$ $\rightarrow 6 s$ transitions of excited Xe neutrals. The transition from tetramers to trimers at $N=40$ and from trimer to dimer at $N=400$ can be explained by a model which takes into account the polarizability, the size of the ionic core and the dissociation energies of the ionic cores. The dimer emission is identified as the $2(1 / 2)_{u} \rightarrow 1(3 / 2)_{g}$ transitions of different vibrational levels. The blue shift of line positions is 30 $( \pm 1) \mathrm{meV}$ compared to those of free molecular Xe ions. This is due to the interaction of the surrounding neutral cluster atoms acting on the embedded ionic Xe molecule.

\section{ACKNOWLEDGMENT}

The financial support for this work was provided by the Deutsche Forschungsgemeinschaft under Grant No. Mo719/ 1-2 and is gratefully acknowledged.

${ }^{1}$ M. Amarouche, G. Durand, and J. P. Malrieu, J. Chem. Phys. 88, 1010 (1987).

${ }^{2}$ H. Haberland, B. v. Issendorf, T. Kolar, H. Kornmeier, C. Ludewigt, and A. Risch, Phys. Rev. Lett. 67, 3290 (1991).

${ }^{3}$ N. Schwentner, E. E. Koch, and J. Jortner, Electronic Excitations in Condensed Rare Gases (Springer-Verlag, Berlin, 1985).

${ }^{4}$ J. Seong, K. C. Janda, N. Halberstadt, and F. Spiegelmann, J. Chem. Phys. 109, 10873 (1998).

${ }^{5}$ M. Grigorov and F. Spiegelmann, Surf. Rev. Lett. 3, 211 (1996).

${ }^{6}$ M. E. Garcia, Phys. Rev. B 48, 8388 (1993).

${ }^{7}$ I. Last and T. F. Georg, J. Chem. Phys. 93, 8925 (1990).

${ }^{8}$ M. Daskalopoulou, H.-U. Böhmer, and S. D. Peyerimhoff, Z. Phys. D: At., Mol. Clusters 15, 161 (1990).

${ }^{9}$ H. Haberland, A. Hofmann, and B. v. Issendorf, J. Chem. Phys. 103, 3450 (1995).

${ }^{10}$ H. Haberland, Surf. Sci. 156, 305 (1985).

${ }^{11}$ A. V. Kanaev, M. C. Castex, L. Museur, R. v. Pietrowski, and T. Möller, Phys. Rev. Lett. 75, 2674 (1995).

${ }^{12}$ R. Karnbach, M. Joppien, J. Stapelfeldt, J. Wörmer, and T. Möller, Rev. Sci. Instrum. 64, 2838 (1993).

${ }^{13}$ O. F. Hagena, Phys. Fluids 17, 894 (1974); Surf. Sci. 106, 101 (1981); Z. Phys. D: At., Mol. Clusters 4, 291 (1987).

${ }^{14}$ U. Buck and R. Krohne, J. Chem. Phys. 105, 5408 (1996).

${ }^{15}$ T. Laarmann, K. v. Haeften, H. Wabnitz, and T. Möller (unpublished).

${ }^{16}$ A. A. Radzig and B. M. Smirnov, Reference Data on Atoms, Molecules, and Ions (Springer-Verlag, Heidelberg, 1985).

${ }^{17}$ K. von Haeften, A. R. B. de Castro, M. Joppien, L. Moussavizade, R. v. Pietrowski, and T. Möller, Phys. Rev. Lett. 78, 4371 (1997).

${ }^{18}$ T. Möller, K. von Haeften, T. Laarmann, and R. v. Pietrowski, Eur. Phys. J. D 9, 5 (1999).

${ }^{19}$ T. Laarmann, diploma thesis, Universität Hamburg, 1998.

${ }^{20}$ T. Kloiber and G. Zimmerer, Phys. Scr. 41, 962 (1990).

${ }^{21}$ P. Stampfli and K. H. Bennemann, Phys. Rev. A 38, 4431 (1988).

${ }^{22}$ G. Ganteför, G. Bröker, E. Holub-Krappe, and A. Ding, J. Chem. Phys. 91, 7972 (1989).

${ }^{23}$ M. Hufnagel, diploma thesis, Universität Hamburg, 1994.

${ }^{24}$ M. Kink, R. Kink, V. Kisand, J. Maksimov, and M. Selg, Nucl. Instrum. Methods Phys. Res. B 122, 668 (1997).

${ }^{25}$ Y. Lu, Y. Morioka, T. Matsui, T. Tanaka, and H. Yoshij, J. Chem. Phys. 102, 1553 (1995).

${ }^{26}$ B. v. Issendorf, A. Hofmann, and H. Haberland, J. Chem. Phys. 111, 2513 (1999).

${ }^{27}$ G. Scoles and K. K. Lehmann, Science 287, 2429 (2000).

${ }^{28}$ R. H. Lipson, P. E. LaRocque, and B. P. Stoicheff, Opt. Lett. 9, 402 (1984).

${ }^{29}$ G. Herzberg, Molecular Spectra and Molecular Structure. I. Spectra of Diatomic Molecules, 2nd ed. (Van Nostrand, New Jersey, 1967), p. 142.

${ }^{30}$ D. E. Freemann, K. Yoshino, and Y. Tanaka, J. Chem. Phys. 61, 4880 (1974).

${ }^{31}$ D. A. Kirkwood, C. A. Woodward, A. Mouhandes, A. J. Stace, A. Bastida, J. Zuniga, A. Requena, and F. X. Gadéa, J. Chem. Phys. 113, 2175 (2000).

${ }^{32}$ A. B. Jones, P. R. Jukes, and A. J. Stace, J. Chem. Phys. 111, 959 (1999).

${ }^{33}$ A. J. Stace, J. Chem. Phys. 93, 6502 (1990).

${ }^{34}$ J. A. Smith, N. G. Gotts, J. F. Winkel, C. A. Woodward, A. J. Stace, and B. J. Whitaker, J. Chem. Phys. 97, 397 (1992).

${ }^{35}$ O. Björneholm, F. Federmann, F. Fössing, and T. Möller, J. Chem. Phys. 104, 1846 (1996).

${ }^{36}$ M. Born, Z. Phys. 1, 45 (1920). 